\title{
Effect of different phosphate binders on fibroblast growth factor 23 levels in patients with chronic kidney disease: a systematic review and meta-analysis of randomized controlled trials
}

\author{
Si-Jie Zhao ${ }^{1 \#}$, Zi-Xuan Wang ${ }^{1 \#}$, Li Chen ${ }^{1}$, Fei-Xuan Wang ${ }^{2}$, Ling-Dong Kong $^{1}$ \\ ${ }^{1}$ School of Life Sciences, Nanjing University, Nanjing, China; ${ }^{2}$ Nanjing Institute of Product Quality Inspection, Nanjing, China \\ Contributions: (I) Conception and design: FX Wang, LD Kong; (II) Administrative support: FX Wang, LD Kong; (III) Provision of study materials \\ or patients: None; (IV) Collection and assembly of data: ZX Wang, SJ Zhao, L Chen; (V) Data analysis and interpretation: SJ Zhao, ZX Wang, FX \\ Wang; (VI) Manuscript writing: All authors; (VII) Final approval of manuscript: All authors. \\ \#These authors contributed equally to this work. \\ Correspondence to: Dr. Ling-Dong Kong. School of Life Sciences, Nanjing University, Nanjing 210023, China. Email: kongld@nju.edu.cn; Mr. \\ Fei-Xuan Wang. Nanjing Institute of Product Quality Inspection, Nanjing 210019, China. Email: cyruswangc@gmail.com.
}

\begin{abstract}
Background: Serum intact fibroblast growth factor 23 (FGF23) levels are progressively increased in relation to the severity of kidney dysfunction. High serum intact FGF23 concentration is associated with the increased cardiovascular morbidity and mortality in patients with chronic kidney disease (CKD). Clinically, phosphate binders are commonly used to reduce serum intact FGF23 levels in CKD patients by lowering serum phosphate levels. It is not clear whether all kinds of phosphate binders can reduce serum intact FGF23 levels, or which kind of phosphate binders is more effective in reducing serum intact FGF23 levels in patients with CKD. The aim of this systematic review and meta-analysis was to compare the efficiency of different kinds of commonly used phosphate binders on serum intact FGF23 levels in patients with CKD.
\end{abstract}

Methods: Systematic searches were performed through PubMed, Cochrane Central Register of Controlled Trials and Embase from 1999 to 2020 . We included the studies performed only in human subjects. All randomized clinical trials (RCTs) were included. Reviews, case reports, letters, commentaries, abstracts and unpublished articles were excluded. Risk of bias was assessed by the Cochrane Collaboration's tool. Random effect was performed in meta-analysis. Meta-regression was used to investigate heterogeneity.

Results: Of 1,895 articles, 15 RCTs (comprising 1,098 participants) were included. Common sources of bias were selection bias. Phosphate binders could reduce serum intact FGF23 levels in patients with CKD [standard mean difference (SMD) of total change in serum intact FGF23 levels was $0.91 \mathrm{PG} / \mathrm{mL}$ (95\% confidence interval: 0.38 to $1.44 \mathrm{PG} / \mathrm{mL}$ ]. Meta-regression explained $89.02 \%$ of heterogeneous sources, indicating that dietary phosphate intake could weaken the effect of phosphate binders on reducing serum intact FGF23 levels, and the effect of phosphate binders on reducing serum intact FGF23 levels in dialysis patients was better than that in early-to-middle CKD patients.

Discussion: Phosphate binders can effectively reduce serum intact FGF23 levels in CKD patients, and iron-based phosphate binders have better effect on reducing serum intact FGF23 levels than other phosphate binders.

Keywords: Fibroblast growth factor 23 (FGF23); phosphate binders; chronic kidney disease (CKD); meta-analysis

Submitted Jul 15, 2021. Accepted for publication Oct 15, 2021.

doi: 10.21037/apm-21-1943

View this article at: https://dx.doi.org/10.21037/apm-21-1943

(c) Annals of Palliative Medicine. All rights reserved. 


\section{Introduction}

Chronic kidney disease (CKD) has become a serious public health problem in recent years (1). In clinical diagnosis of $\mathrm{CKD}$, serum creatinine, as well as decomposition products of creatinine and creatine phosphate are generally used as biomarkers to judge kidney dysfunction (1-5). Currently, many clinical observations and animal experiments show that serum intact fibroblast growth factor 23 (FGF23) is a sensitive biomarker for CKD for it rises earlier than most other serum parameters, including serum creatinine and creatine phosphate $(6,7)$. Phosphaturic hormone FGF23, derived and secreted primarily by bone osteocytes, is a $32-\mathrm{kDa}$ glycoprotein (8). The key role of intact FGF23 is to maintain phosphate homeostasis (9). Serum intact FGF23 levels are progressively increased in relation to the severity of kidney function decline in CKD patients $(2,7,9,10)$. In face of reduced nephron mass, intact FGF23 production is increased to counteract chronic phosphate retention by promoting urinary phosphate excretion (11). In addition, inflammatory factor tumor necrosis factoralpha also stimulates the production of plasma FGF23 levels in mouse model of CKD (12). In patients on hemodialysis, there is a $100-1,000$-fold increase in serum intact FGF23 levels (13). Of note, a study in USA shows that there is a close relationship between serum level of intact FGF23 and the prevalence of cardiac events, including myocardial infarction, heart failure, and left ventricle hypertrophy (14). High level of FGF23 has been proven to increase the possibility of aortic and coronary calcification (15) and cause endothelial dysfunction to cardiovascular diseases $(16,17)$. Therefore, high serum intact FGF23 concentration is associated with the increased cardiovascular morbidity and mortality in patients with CKD $(10,13,18,19)$.

Reduction of high intact FGF23 concentration is a strategy, possibly improving the consequences of CKD. The most common way of reducing serum intact FGF23 concentration is through decreasing serum levels of phosphate, which can be achieved by a phosphate-restricted diet or the administration of phosphate binders (20). In the end stage of CKD, only using phosphate-restricted diet fails to control hyperphosphatemia $(19,21)$, whereas, phosphate binders have been shown to significantly reduce serum phosphorus levels, suggesting that using phosphate binders is necessary (20,22-24). The commonly used phosphate binders include calcium carbonate, calcium acetate, lanthanum carbonate, sevelamer and ferric citrate (25). However, which kind of phosphate binders is more effective in reducing serum intact FGF23 levels in patients with CKD, different studies show inconsistent results (26). A study comparing three kinds of phosphate binders with placebo, shows that only in patients treated with sevelamer carbonate, serum intact FGF23 levels are significantly decreased (27). Yet an 8-week study shows an increase of serum intact FGF23 levels in patients treated with calcium acetate (28). Therefore, it is necessary to analysis the reliable data, specifically focusing on reduction of serum intact FGF23 levels by phosphate binders.

The aim of our meta-analysis was to compare the efficiency of different kinds of commonly used phosphate binders on reducing serum intact FGF23 levels in patients with CKD. We present the following article in accordance with the PRISMA reporting checklist (available at https://apm. amegroups.com/article/view/10.21037/apm-21-1943/rc).

\section{Methods}

Meta-analysis was conducted according to the Cochrane handbook (29).

\section{Data sources and searches}

We searched published English-language researches in PubMed, Cochrane Central Register of Controlled Trials and Embase from 1999 to 2020. We adjusted the search strategies to meet the specifications of the individual databases (Table S1). References of selected retrieved articles were also examined.

\section{Inclusion and exclusion criteria}

The inclusion criteria were independently applied to all identified studies. We included the studies performed only in human subjects. Published randomized clinical trials (RCTs) that compared a phosphate binder with control group in CKD patients were included in this study. We included participants with CKD stages 3 to 5 , which are defined as estimating glomerular filtration rate less than $60 \mathrm{~mL} / \mathrm{min}$ per $1.73 \mathrm{~m}^{2}$. We excluded studies without measuring serum intact FGF23 levels before and after the treatment. Reviews, case reports, letters, commentaries, abstracts and unpublished articles were excluded.

\section{Data extraction}

Two researchers independently reviewed titles and abstracts 
of the first 30 records and discussed inconsistencies until the consensus was obtained. Then the researchers independently screened titles and abstracts of all records retrieved. In case of disagreement, the consensus on which full-text articles were screened was reached by discussion. We designed data extraction form, and two researchers used this form to extract data from eligible studies independently. Extracted data were compared, with any discrepancies being resolved by discussion. We searched papers with fulltext versions and identified them in the initial screening. Study designs, participant characteristics, interventions, and outcome measures were extracted. We contacted the authors of the primary reports to request any unpublished data. If numeric outcome data were unavailable, we used webplotDigitizer (Version 4.4) to extract the data from figures and graphs.

\section{Assessment of bias risk}

Assessment of bias risk was performed independently by two researchers. All disagreements were resolved by consensus. We conducted a subjective assessment of methodological quality for RCT studies by the Cochrane Collaboration's tool for assessing risk of bias (29). In this assessment, each component was categorized as having a high, low or unclear risk of bias: random sequence generation, allocation concealment, blinding of participants and personnel, blinding of outcome assessment, incomplete outcome data, and selective reporting.

\section{Statistical analysis}

We reported the standard mean difference (SMD) of the change in serum intact FGF23 concentration before and after the treatment of phosphate binders. We classified the direction of effect as 'favours placebo' or 'favours phosphate binder'. We excluded the studies from the analysis if they did not have enough information required for continuous data comparison. Serum intact FGF23 levels measured in most of these studies were expressed in the form of interquartile range (IQR). We estimated the mean and the variance for such trials according to the available data. If the sample size is larger than 25 , we used the median to estimate mean. The standard deviation could be estimated using the median, low and high end of the range, and sample size. If the sample size is between 15 and 70 , the formula range/4 was the best estimator for the standard deviation. If the sample size is larger than 70 , the formula range/6 gave the best estimator for the standard deviation (30).

We used the Q statistic and $\mathrm{I}^{2}$ statistic to quantify heterogeneity of effect size estimates across these studies. For $\mathrm{Q}$ statistic, substantial heterogeneity was defined as $\mathrm{P}<0.1$. For $\mathrm{I}^{2}$ statistic, $\mathrm{I}^{2}<25 \%$ represented low heterogeneity, $\mathrm{I}^{2}=25-50 \%$ represented moderate heterogeneity, $\mathrm{I}^{2}>50 \%$ represented substantial heterogeneity (31). If $\mathrm{I}^{2}$ was $<50 \%$, the heterogeneity was acceptable, and the fixed effect model was used for the analysis. If $\mathrm{I}^{2}$ was greater than $50 \%$, the heterogeneity was high, and a random effect model was used for the analysis. Meta-regression was used to investigate heterogeneity. And we used subgroup analysis instead of sensitivity analysis to assess robustness of the synthesized results.

Possible publication bias was assessed using funnel plot and Begg's regression test $(\mathrm{P}<0.05$ was considered significant) (29). The data analysis was performed using Stata 14 and RevMan 5 (32).

\section{Results}

\section{Description of included studies}

We initially searched 1,895 records through database searching and citation searching, 465 duplicate records were removed and 1,303 records were excluded by title and abstract. Of the 127 reports initially screened, 11 reports were not retrieved, and the remaining 116 reports were undergone full-length review. After reviewing, 101 reports were excluded for they were review, or not RCT studies using placebo control, or meet other exclusion criteria. Finally, we included 15 studies for analysis (Figure 1).

Data were extracted from 15 studies involving total of 1,098 CKD participants for qualitative analysis. The included studies varied in sample size (21 to 155), type and dosage of phosphate binders used, and duration of the treatment (4 weeks to 12 months). The characteristics of the 15 included studies were outlined in Table 1 .

\section{Risk of bias in included studies}

All included studies were assessed for risk of bias using the Cochrane Collaboration's tool, and the results were shown in Figure 2, Table 2 and Figure S1.

\section{Meta-analysis results}

Out of 15 studies, 13 studies compared the effect of 


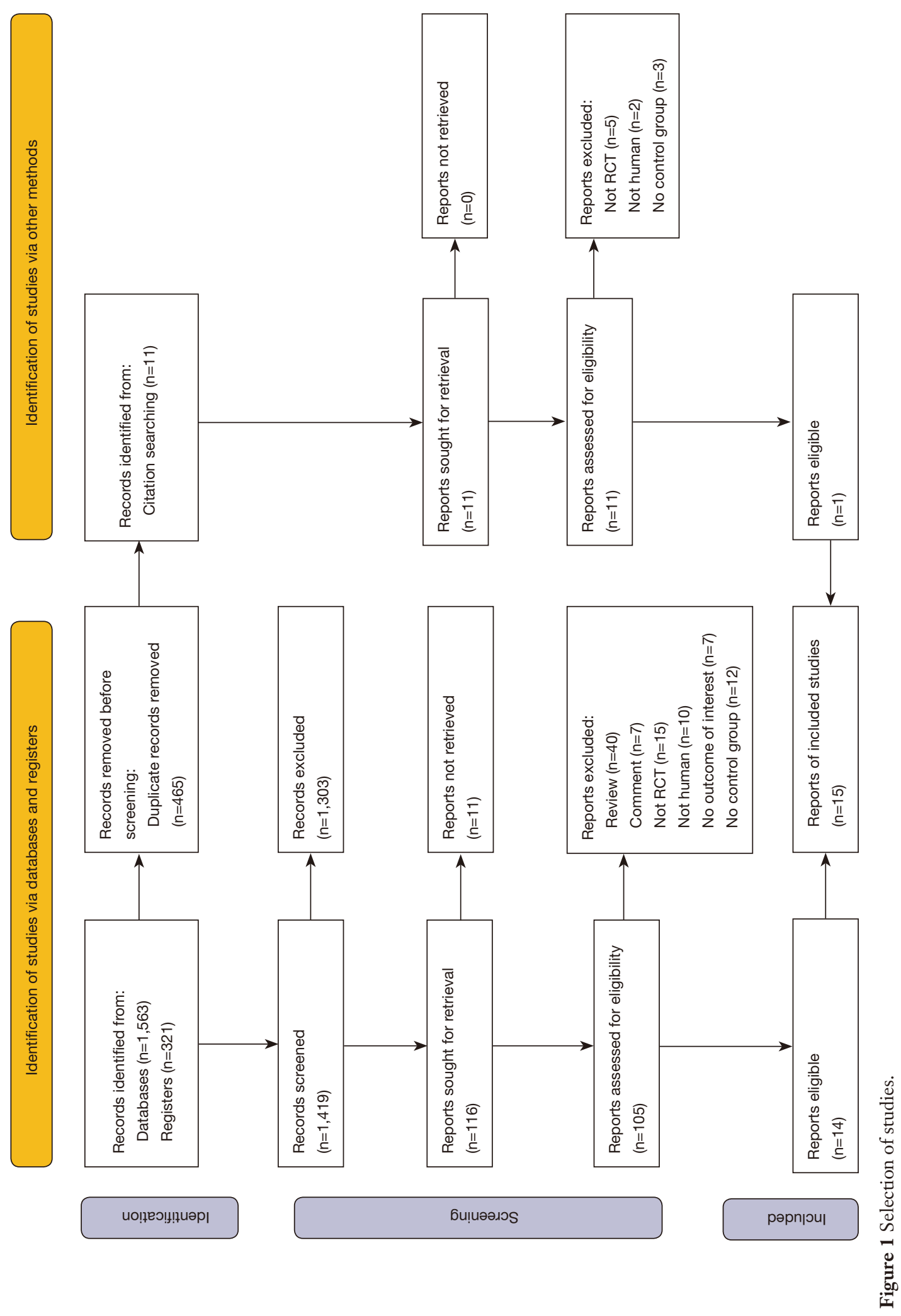




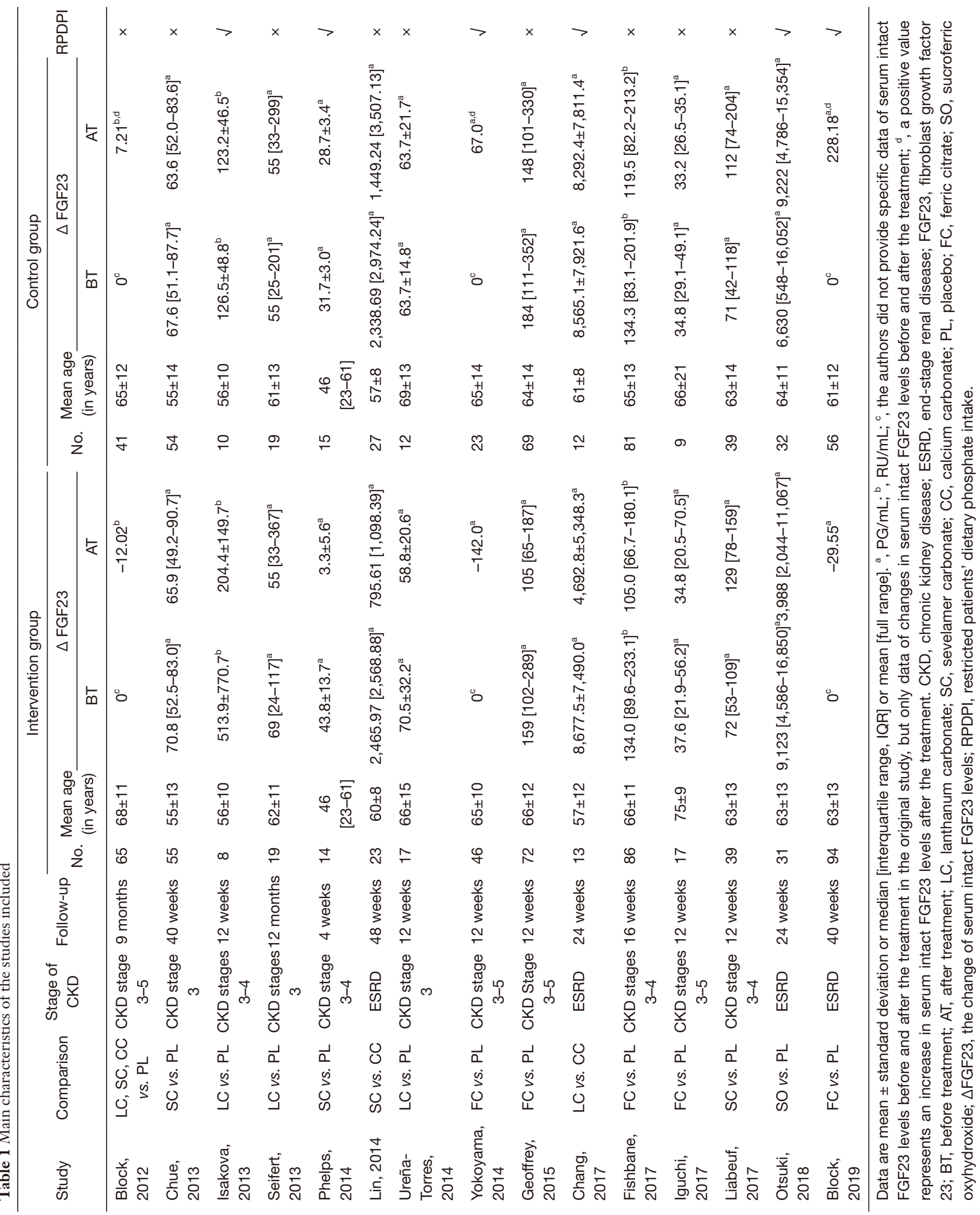




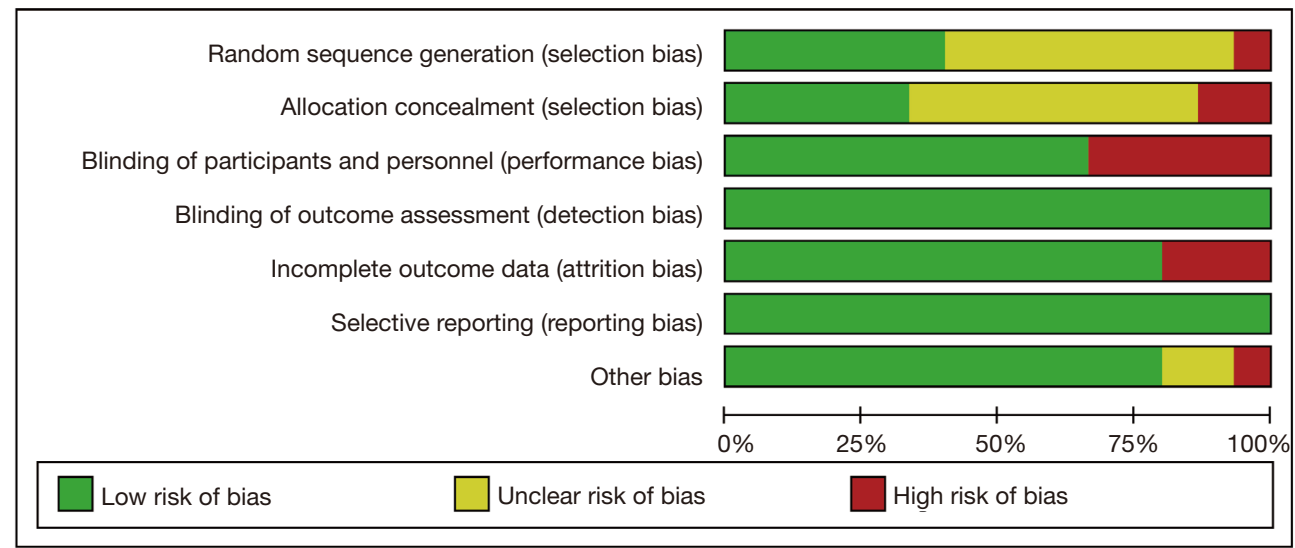

Figure 2 Risk of bias graph: each risk of bias item presented as percentages across all included studies.

different phosphate binders and placebo on serum intact FGF23 levels (27,33-44). Other two studies compared the effect of non-calcium-based phosphate binder and calciumbased phosphate binder on serum intact FGF23 levels $(45,46)$. Thirteen studies containing phosphate binders group and placebo group were included to analyze the effect of phosphate binders on serum intact FGF23 levels in patients with CKD (27,33-44). Among of them, eight studies showed that phosphate binders could reduce serum intact FGF23 levels in patients with CKD (Geoffrey A 2015, Otsuki T 2018, Isakova T 2013, Fishbane S 2017, Seifert ME 2013, Block GA 2012, Yokoyama K 2014, Block GA 2019) $(27,33,37-42)$. Other five studies showed that phosphate binders did not reduce serum intact FGF23 levels in patients with CKD (Chue CD 2013, Ureña-Torres P 2014, Iguchi A 2017, Liabeuf S 2017, Phelps KR 2014) $(34-36,43,44)$.

More information for main characteristics of the included studies was shown in Table 1. Taken together, we used a randomized effect model to investigate the effect of phosphate binders on serum intact FGF23 levels of CKD patients. There was significant difference in the change of serum intact FGF23 levels between experimental group and control group (Figure 3). The SMD of total changes in serum intact FGF23 levels was $0.91 \mathrm{PG} / \mathrm{mL}$ (95\% CI: 0.38 to $1.44, \mathrm{P}<0.05)$, suggesting that phosphate binders could reduce serum intact FGF23 levels in patient with CKD. We found that the heterogeneity between different studies was high $\left(I^{2}=93.1 \%, \operatorname{tau}^{2}=0.82\right)$. In order to make the results of the meta-analysis more convincing, we would further discuss the sources of heterogeneity in the meta-regression analysis.

\section{Subgroup analysis}

To further investigate the effect of different phosphate binders on reducing serum intact FGF23 levels in patients with CKD, we performed a subgroup analysis (Figure 4). Six studies used iron-based phosphate binders to treat patients with CKD $(33,36,37,39,41,42)$, while 7 studies used noniron-based phosphate binders to treat patients with CKD $(27,34,35,38,40,43,44)$. There was a significant statistical difference, indicating that the effect of ferric citrate on decreasing serum intact FGF23 levels was better than noiron phosphate binders $(\mathrm{P}<0.05)$.

Two studies compared the effects of calcium-based phosphate binder and non-calcium-based phosphate binder on reducing serum intact FGF23 levels in patient with CKD $(45,46)$. We performed a meta-analysis to investigate the effects of phosphate binders in serum intact FGF23 levels between calcium-based phosphate binder and noncalcium-based phosphate binder (Figure 5). There was no significant statistical difference between calcium-based phosphate binder group and non-calcium-based phosphate binder group $(\mathrm{P}<0.05)$.

\section{Meta-regression}

Meta-analysis results showed that there was statistical between-study heterogeneity $\left(\mathrm{I}^{2}=93.1 \%, \mathrm{P}=0.968\right.$, $\left.\operatorname{tau}^{2}=0.82\right)$. In order to find the source of heterogeneity, we have reviewed the treatment modality, as well as the inclusion and exclusion criteria for CKD patients from each study. We noted that there were two possible sources of heterogeneity. One was whether the patient's dietary 


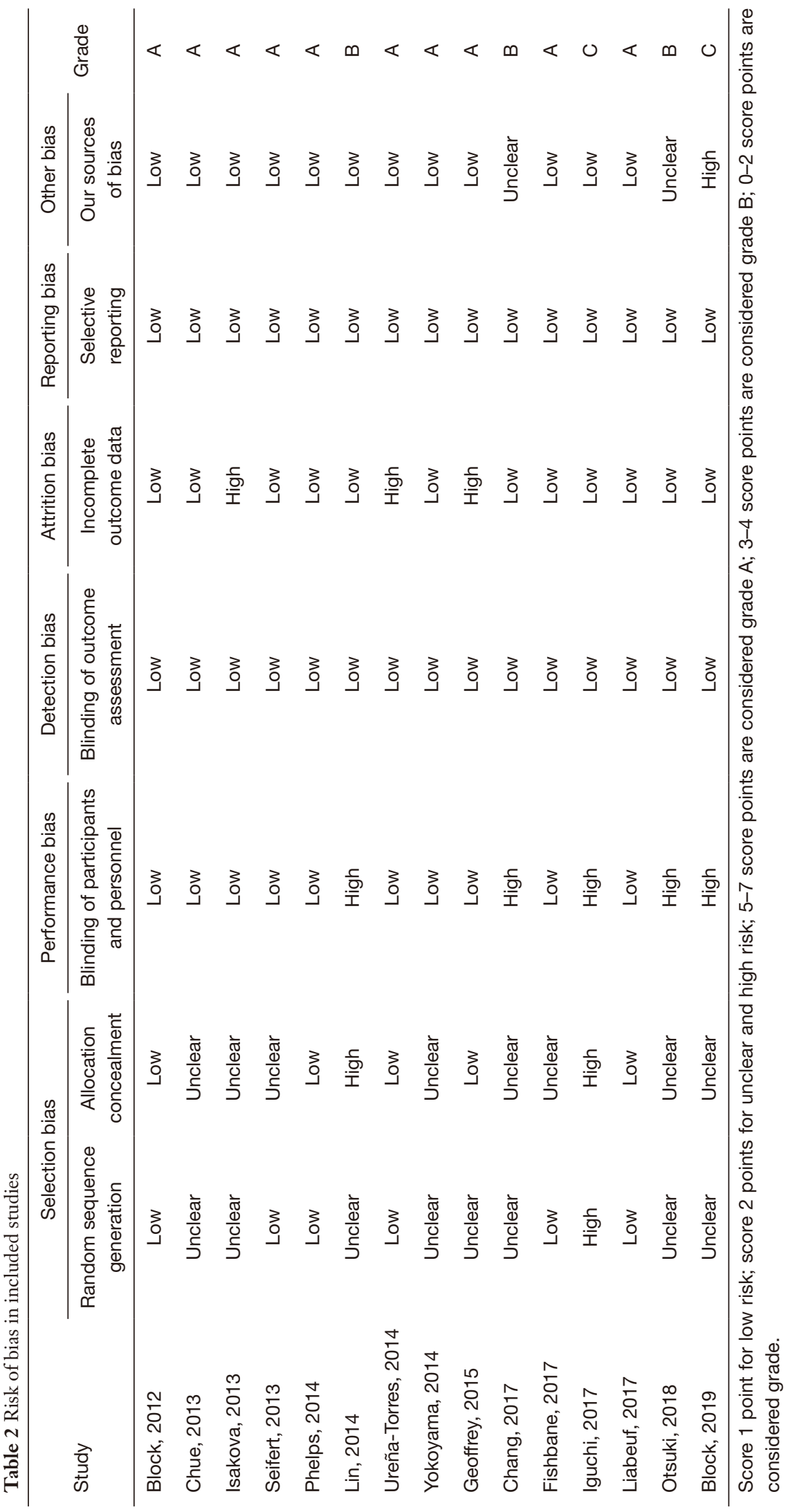




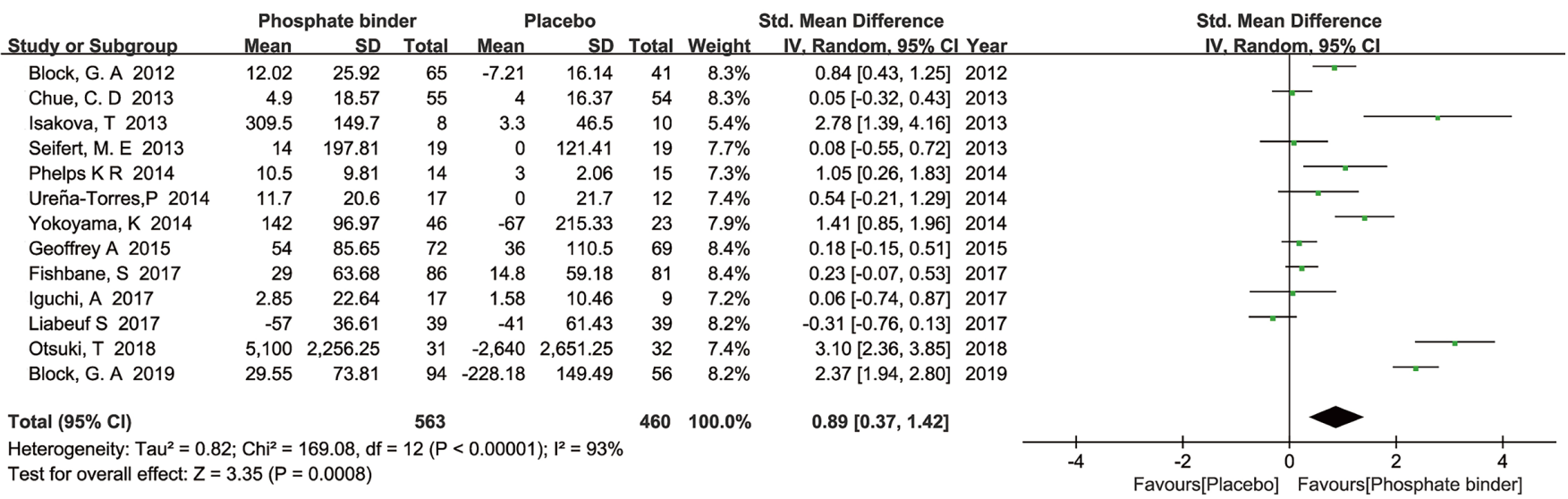

Figure 3 Comparison of serum intact fibroblast growth factor 23 (FGF23) between phosphate binders and control group in patients with chronic kidney disease (CKD). Meta-analysis pooling of standard mean differences by the method of Cohen, using the random-effects inverse-variance model with DerSimonian-Laird estimate of $\operatorname{tau}^{2}, \mathrm{I}^{2}=$ proportion of total variation in effect estimate due to between-study heterogeneity (based on Q).

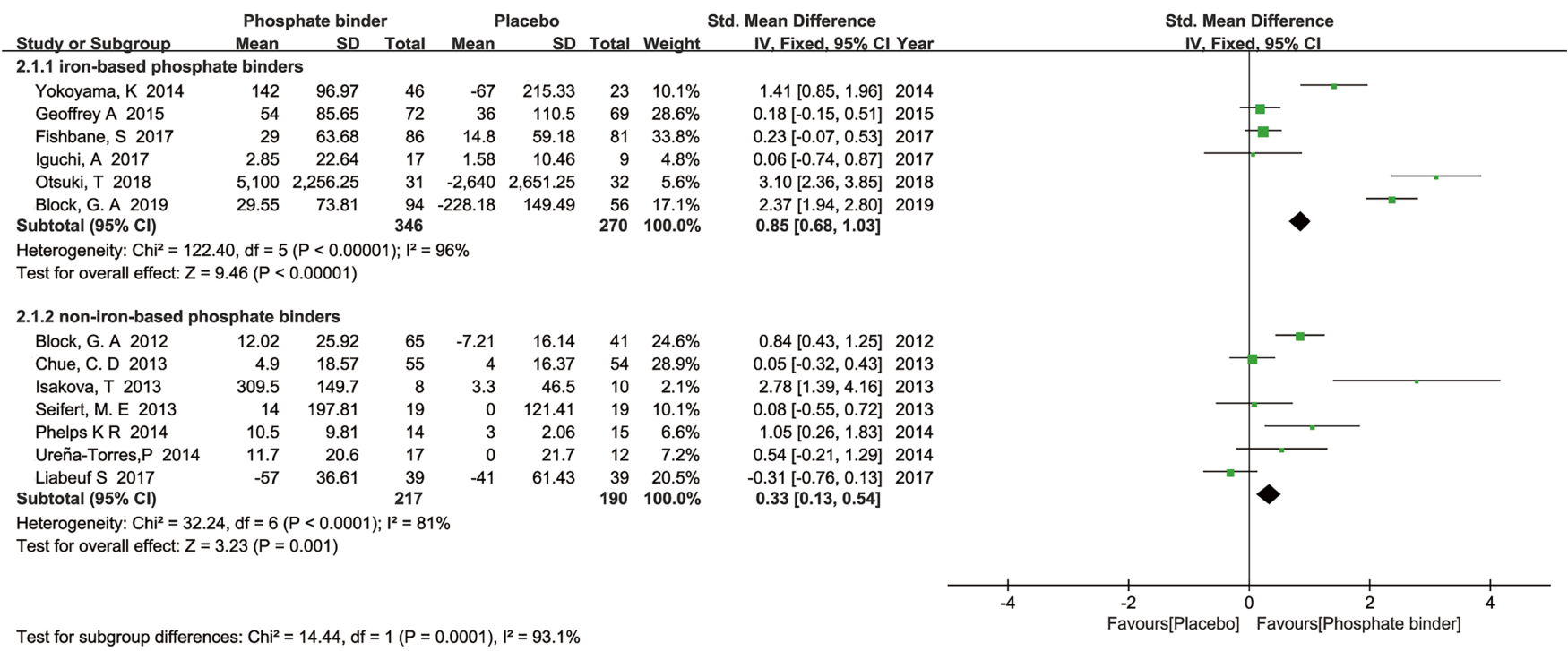

Figure 4 Subgroup analysis of serum intact fibroblast growth factor 23 (FGF23) levels between iron-based phosphate binder and non-iron phosphate binder in patients with chronic kidney disease (CKD).

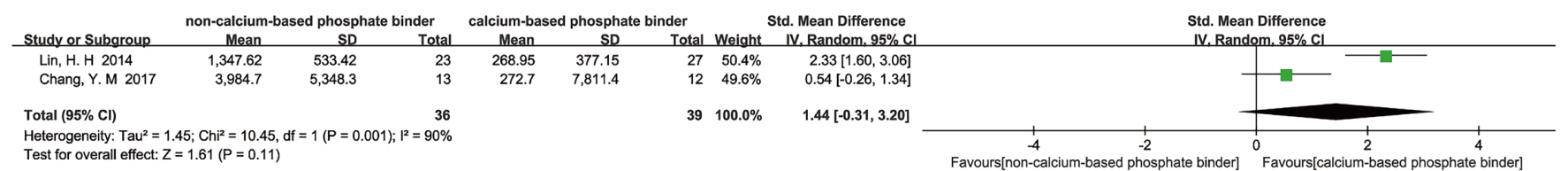

Figure 5 Meta-analysis of serum intact fibroblast growth factor 23 (FGF23) between calcium-based phosphate binder and non-calciumbased phosphate binder in patients with chronic kidney disease (CKD). 


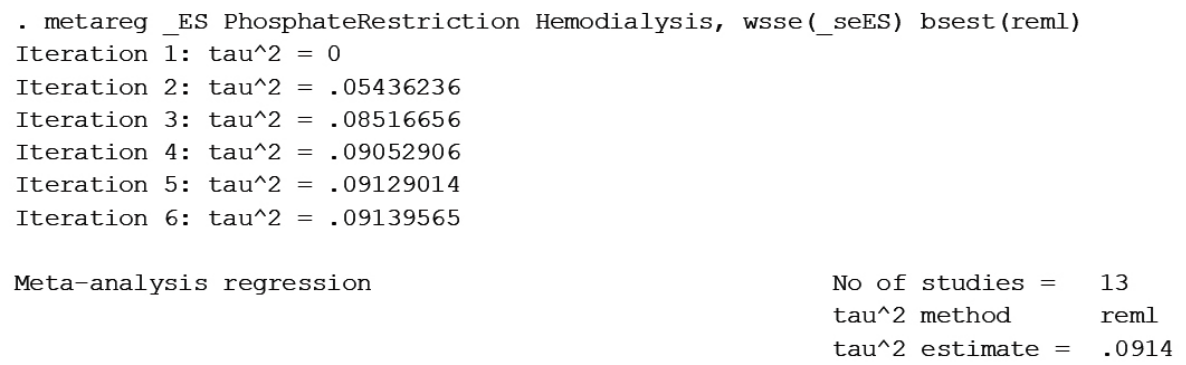

Successive values of tau^ 2 differ by less than $10^{\wedge}-4$ :convergence achieved

\begin{tabular}{r|rrrrrr}
\hline & Coef. & Std. Err. & $z$ & P $>|z|$ & [95\% Conf. Interval] \\
\hline PhosphateR n & 1.315569 & .3254226 & 4.04 & 0.000 & .6777529 & 1.953386 \\
Hemodialysis & 1.135557 & .4174113 & 2.72 & 0.007 & .3174455 & 1.953668 \\
_Cons & .2107677 & .1382926 & 1.52 & 0.127 & -.0602809 & .4818163 \\
\hline
\end{tabular}

Figure 6 Meta regression analysis results. PhosphateR n, whether dietary phosphorus intake was restricted in patients with chronic kidney disease (CKD); hemodialysis, whether patients with CKD performed hemodialysis.

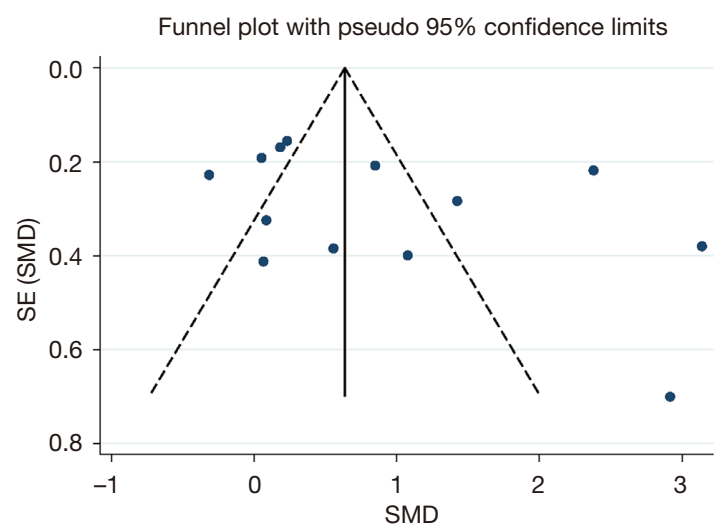

Figure 7 Funnel plot to assess publication bias for serum intact fibroblast growth factor 23 (FGF23) levels in patients with chronic kidney disease (CKD).

phosphorus intake was restricted. There were 4 studies restricted patient's dietary phosphate intake $(37,38,41,42)$, while 9 studies did not restrict patient's dietary phosphate intake during the treatment $(27,33-36,39,40,43,44)$. The other was whether patients performed hemodialysis. There were 2 studies included end-stage CKD patients receiving hemodialysis $(37,42)$, while 11 studies included early-to-middle stage CKD patients who did not receive hemodialysis (33-36,38-41,43,44). We used whether patient's dietary phosphorus intake was restricted as well as whether patients performed hemodialysis as covariates made meta-regression analysis (Figure 6). We found that the $\operatorname{tau}^{2}$ value was reduced from 0.82 to 0.09 , explaining $89.02 \%$ of heterogeneous sources, indicating that dietary phosphorus intake restrict and hemodialysis were the main factors affecting the effect of phosphate binders on reducing serum intact FGF23 levels in patient with CKD. Dietary phosphate intake could weaken the effect of phosphate binders on reducing serum intact FGF23 levels. The effect of phosphate binders on reducing serum intact FGF23 levels in dialysis patients was better than that in early-tomiddle CKD patients.

\section{Publication bias}

To investigate potential publication bias, we examined a Begg's funnel plot of the included studies in assessing total changes in serum intact FGF23 levels of CKD patients. From this plot (Figure 7), bias was not presented because there was symmetrical distribution of studies on both sides of the mean.

\section{Discussion}

Our meta-analysis showed that phosphate binders could reduce serum levels of both intact FGF23 and phosphorus in patients with CKD. We considered that serum intact FGF23 reduction was not only caused by phosphorus concentration reduction, but also related to other effect of different phosphorus binders themselves. Study has shown that iron lack stimulates the synthesis of intact FGF23 in 
patients with CKD (47). Therefore, we considered that iron-based phosphate binder has also supplemented iron elements during reducing phosphate, further inhibiting the formation of intact FGF23, which possibly explains why iron-based phosphate binder is better in patients with CKD.

In this study, we did not control the duration of phosphate binders. Under the strict inclusion and exclusion criteria, the meta-analysis itself included fewer studies. If the duration is controlled, the sample size may be further reduced, and the credibility of the meta-analysis results may be greatly decreased. We carefully reviewed each original study, but many studies only report baseline and outcome data. Therefore, it is difficult to obtain intermediate data. On the other hand, we did not control the exact dosage of phosphate binders, but the dosage was within a similar range in included studies. The reason is that when phosphate binders are used to improve the consequences of CKD, their dosages depend on serum phosphorus concentration in patients. In the original study, the dosage of phosphate binders was different according to the state of the patients. The dosage is decreased when phosphate binders cause side effect, and is increased during high serum phosphorus concentration in each study (Table 3). Generally, the dosage of phosphate binders is relatively consistent for CKD patients with similar serum phosphorus concentration, for example, the dosage of lanthanum carbonate is usually $3 \mathrm{~g} / \mathrm{d}$ for patients with CKD stages 3 to $5(48,49)$. Since only patients with CKD stages 3 to 5 were included in our study, the dosage change of phosphate binders between different studies was small, which further weakened the effect of the dosage on the meta-analysis results. Thus, the credibility of our meta-analysis was relatively high.

Intact FGF23 is suggested to maintain phosphate homeostasis (9). In the kidney, physiological function of intact FGF23 is mediated by binding to the complex of fibroblast growth factor receptor (FGFR) and its specific coreceptor klotho (50). Intact FGF23 forms FGF23-FGFRKlotho complex with FGFR and Klotho, which can inhibit expression levels of sodium-dependent phosphate transport protein $2 \mathrm{~A}$ and sodium-dependent phosphate transport protein $2 \mathrm{C}$ at proximal renal tubules, thereby promote renal phosphate excretion (51-53). Furthermore, FGF23-FGFRKlotho complex reduces serum 1,25-dihydroxyvitamin D3 levels by down-regulating renal expression of cytochrome P450 family 27 subfamily B member 1 encoding $1 \alpha$-hydroxylase, which inhibits the conversion of prohormone 25-hydroxyvitamin D3 into its active form, as well as by up-regulating cytochrome P450 family 24 subfamily
A member 1, which encodes catabolic 24-hydroxylase (54). Decrease of renal 1,25-dihydroxyvitamin D3 production causes a low abundance of type IIb sodium-phosphate cotransporter (NPT2b) in gastrointestinal tract, thereby limiting phosphate uptake $(52,55)$.

Phosphate binders reduce serum phosphate levels, subsequently, up-regulates NPT2b-dependent dietary phosphate absorption (56-59). Thus, the effectiveness of phosphate binders may be offset by the contribution of NPT2b up-regulation to total intestinal phosphate absorption, possibly explaining why dietary phosphate intake could weaken the effect of phosphate binders on reducing serum intact FGF23 levels in CKD patients.

There is a positive correlation between serum phosphorus level and FGF23 level, but the specific regulatory mechanism has not been clearly explained in the existing studies. In addition, the mechanism by which how dietary phosphorus intake 'signal' is transmitted to FGF23 change has not been clearly explained. The regulation of FGF23-mdiated homeostasis in human body is relatively complex, with a multi-pathway of regulatory network. Future research direction can start from the elaboration of these mechanisms.

Of note, some of those phosphate binders bring different side effects. Calcium acetate has more severe gastrointestinal side effects than calcium carbonate. High dose of calcium-based phosphate binders is reported to increase the risk of hypercalcaemic and vascular calcification in patients with CKD (60). Compared with calcium salts, sevelamer shows equivalent phosphate control and less hypercalcaemic events in CKD patients (60). As with all other phosphate binders, sevelamer has gastrointestinal side effects $(61,62)$. Lanthanum, a trivalent cation that binds phosphate ionically, is active over a wide $\mathrm{pH}$ (63). Lanthanum has been shown in clinical study to be an effective, well-tolerated phosphate binder with no evidence of significant side effects in patients with CKD (64). Ferric citrate is an intestinal phosphate binder, which stores iron, increases hemoglobin levels, and reduces serum phosphate levels in patients with end-stage renal disease undergoing hemodialysis (33). Therefore, in the clinical treatment of CKD, different phosphate binders should be selected according to the patient's situation to prevent side effects.

The strengths of this study lie in its objective assessment of study quality and rigorous methodology, which conducted according to the Cochrane handbook. Despite these strengths, the systematic review and meta-analysis have limitations, both because of the limitations of the 
Table 3 Duration and dosage of phosphate binders in each study

\begin{tabular}{|c|c|c|c|c|c|}
\hline Study & Comparison & \multicolumn{2}{|c|}{ Dosage } & Duration & Note \\
\hline Block, 2012 & LC, SC, CC vs. PL & LC: 3.0, SC: 4.8, CC: 4.0 & $\begin{array}{c}\text { PL: } 3.0, \text { PL: } 4.8 \\
\text { PL: } 4.0\end{array}$ & 9 months & $\begin{array}{l}\text { The dosage was adjusted between } \\
1.5 \text { and } 9.6 \mathrm{~g} / \mathrm{d} \text { according to the target } \\
\text { range of serum phosphate } \\
(3.5-4.5 \mathrm{mg} / \mathrm{dL})\end{array}$ \\
\hline Isakova, 2013 & LC vs. PL & 3.0 & 3.0 & 12 weeks & NA \\
\hline Seifert, 2013 & LC vs. PL & 3.0 & 3.0 & 12 months & NA \\
\hline Ureña-Torres, 2014 & LC vs. PL & 3.0 & 3.0 & 12 weeks & NA \\
\hline Yokoyama, 2014 & FC vs. PL & 3.0 & 3.0 & 12 weeks & $\begin{array}{c}\text { The dosage was adjusted between } \\
1.5 \text { and } 6.0 \mathrm{~g} / \mathrm{d} \text { according to the target } \\
\text { range of serum phosphate } \\
(2.5-4.5 \mathrm{mg} / \mathrm{dL})\end{array}$ \\
\hline Geoffrey, 2015 & FC vs. PL & 3.0 & 3.0 & 12 weeks & Containing $\mathrm{Fe}^{3+}: 0.63 \mathrm{~g} / \mathrm{d}$ \\
\hline Chang, 2017 & LC vs. CC & 1.644 & 3.375 & 24 weeks & NA \\
\hline
\end{tabular}

LC, lanthanum carbonate; SC, sevelamer carbonate; CC, calcium carbonate; PL, placebo; FC, ferric citrate; SO, sucroferric oxyhydroxide; $\mathrm{NA}$, not available; $\mathrm{Fe}^{3+}$, ferric iron.

research we were able to retrieve and the data extraction. We searched in three databases only and focused on studies published in English, and the hand search was limited to references of review studies. These choices resulted in fewer studies included in our meta-analysis and reduced the credibility of the conclusions. Further limitation is accuracy of available data: the measurement of FGF23 levels in most of these studies are expressed in the form of IQR, although we used the available data to estimate the mean and variance in those trials according to the Cochrane handbook for systematic reviews of interventions, there still remains a little error between the estimated value and the real data.

\section{Conclusions}

We find that phosphate binders can effectively reduce serum intact FGF23 levels in CKD patients, which is also affected by other conditions, such as dietary phosphate intake, and iron deficiency-caused anemia. Dietary phosphate intake could weaken the effect of phosphate binders on reducing serum intact FGF23 levels in CKD patients. Especially in patients with anemia caused by iron deficiency, ironbased phosphate binders are more effective on reducing serum intact FGF23 levels than other phosphate binders. These findings may guide the clinical treatment of CKD patients with the best medication methods. For example, 
iron-based phosphate binders should be used when there are complications of anemia caused by iron deficiency, and calcium-based phosphate binders should be avoided when patients have gastrointestinal discomfort.

\section{Acknowledgments}

We would like to acknowledge everyone who has supported and contributed to each cohort included in this study.

Funding: None.

\section{Footnote}

Reporting Checklist: The authors have completed the PRISMA reporting checklist. Available at https://apm. amegroups.com/article/view/10.21037/apm-21-1943/rc

Peer Review File: Available at https://apm.amegroups.com/ article/view/10.21037/apm-21-1943/prf

Conflicts of Interest: All authors have completed the ICMJE uniform disclosure form (available at https://apm. amegroups.com/article/view/10.21037/apm-21-1943/coif). The authors have no conflicts of interest to declare.

Ethical Statement: The authors are accountable for all aspects of the work in ensuring that questions related to the accuracy or integrity of any part of the work are appropriately investigated and resolved.

Open Access Statement: This is an Open Access article distributed in accordance with the Creative Commons Attribution-NonCommercial-NoDerivs 4.0 International License (CC BY-NC-ND 4.0), which permits the noncommercial replication and distribution of the article with the strict proviso that no changes or edits are made and the original work is properly cited (including links to both the formal publication through the relevant DOI and the license). See: https://creativecommons.org/licenses/by-nc-nd/4.0/.

\section{References}

1. Webster AC, Nagler EV, Morton RL, et al. Chronic Kidney Disease. Lancet 2017;389:1238-52.

2. Zhang WR, Parikh CR. Biomarkers of Acute and Chronic Kidney Disease. Annu Rev Physiol 2019;81:309-33.

3. Drawz P, Rahman M. Chronic kidney disease. Ann Intern Med 2015;162:ITC1-16.
4. Andrassy KM. Comments on 'KDIGO 2012 Clinical Practice Guideline for the Evaluation and Management of Chronic Kidney Disease'. Kidney Int 2013;84:622-3.

5. Levey AS, de Jong PE, Coresh J, et al. The definition, classification, and prognosis of chronic kidney disease: a KDIGO Controversies Conference report. Kidney Int 2011;80:17-28.

6. Desjardins L, Liabeuf S, Renard C, et al. FGF23 is independently associated with vascular calcification but not bone mineral density in patients at various CKD stages. Osteoporos Int 2012;23:2017-25.

7. Gutiérrez OM. Fibroblast growth factor 23 and disordered vitamin D metabolism in chronic kidney disease: updating the "trade-off" hypothesis. Clin J Am Soc Nephrol 2010;5:1710-6.

8. Ho BB, Bergwitz C. FGF23 signalling and physiology. J Mol Endocrinol 2021;66:R23-32.

9. Vogt I, Haffner D, Leifheit-Nestler M. FGF23 and Phosphate-Cardiovascular Toxins in CKD. Toxins (Basel) 2019;11:647.

10. Fliser D, Kollerits B, Neyer U, et al. Fibroblast growth factor 23 (FGF23) predicts progression of chronic kidney disease: the Mild to Moderate Kidney Disease (MMKD) Study. J Am Soc Nephrol 2007;18:2600-8.

11. Komaba H, Fukagawa M. FGF23-parathyroid interaction: implications in chronic kidney disease. Kidney Int 2010;77:292-8.

12. Egli-Spichtig D, Imenez Silva PH, Glaudemans B, et al. Tumor necrosis factor stimulates fibroblast growth factor 23 levels in chronic kidney disease and non-renal inflammation. Kidney Int 2019;96:890-905.

13. Jean G, Terrat JC, Vanel T, et al. High levels of serum fibroblast growth factor (FGF)-23 are associated with increased mortality in long haemodialysis patients. Nephrol Dial Transplant 2009;24:2792-6.

14. Faul C, Amaral AP, Oskouei B, et al. FGF23 induces left ventricular hypertrophy. J Clin Invest 2011;121:4393-408.

15. Lu X, Hu MC. Klotho/FGF23 Axis in Chronic Kidney Disease and Cardiovascular Disease. Kidney Dis (Basel) 2017;3:15-23.

16. Silswal N, Touchberry CD, Daniel DR, et al. FGF23 directly impairs endothelium-dependent vasorelaxation by increasing superoxide levels and reducing nitric oxide bioavailability. Am J Physiol Endocrinol Metab 2014;307:E426-36.

17. Adema AY, de Borst MH, Ter Wee PM, et al. Dietary and pharmacological modification of fibroblast growth factor-23 in chronic kidney disease. J Ren Nutr 
2014;24:143-50.

18. Isakova $\mathrm{T}$, Xie $\mathrm{H}$, Yang $\mathrm{W}$, et al. Fibroblast growth factor 23 and risks of mortality and end-stage renal disease in patients with chronic kidney disease. JAMA 2011;305:2432-9.

19. Gutiérrez OM, Mannstadt M, Isakova T, et al. Fibroblast growth factor 23 and mortality among patients undergoing hemodialysis. N Engl J Med 2008;359:584-92.

20. Ruospo M, Palmer SC, Natale P, et al. Phosphate binders for preventing and treating chronic kidney disease-mineral and bone disorder (CKD-MBD). Cochrane Database Syst Rev 2018;8:CD006023.

21. Kestenbaum B. Con: Phosphate binders in chronic kidney disease. Nephrol Dial Transplant 2016;31:189-94.

22. Wang F, Lu X, Zhang J, et al. Effect of Lanthanum Carbonate on All-Cause Mortality in Patients Receiving Maintenance Hemodialysis: a Meta-Analysis of Randomized Controlled Trials. Kidney Blood Press Res 2018;43:536-44.

23. Floege J, Covic AC, Ketteler M, et al. Long-term effects of the iron-based phosphate binder, sucroferric oxyhydroxide, in dialysis patients. Nephrol Dial Transplant 2015;30:1037-46.

24. Sakaguchi Y, Hamano T, Obi Y, et al. A Randomized Trial of Magnesium Oxide and Oral Carbon Adsorbent for Coronary Artery Calcification in Predialysis CKD. J Am Soc Nephrol 2019;30:1073-85.

25. Barreto FC, Barreto DV, Massy ZA, et al. Strategies for Phosphate Control in Patients With CKD. Kidney Int Rep 2019;4:1043-56.

26. Mehta R, Isakova T. Continued Search for Therapies to Favorably Modify Phosphate and FGF23 Levels in CKD. Clin J Am Soc Nephrol 2017;12:1911-3.

27. Block GA, Wheeler DC, Persky MS, et al. Effects of phosphate binders in moderate CKD. J Am Soc Nephrol 2012;23:1407-15.

28. Yilmaz MI, Sonmez A, Saglam M, et al. Comparison of calcium acetate and sevelamer on vascular function and fibroblast growth factor 23 in CKD patients: a randomized clinical trial. Am J Kidney Dis 2012;59:177-85.

29. Cumpston M, Li T, Page MJ, et al. Updated guidance for trusted systematic reviews: a new edition of the Cochrane Handbook for Systematic Reviews of Interventions. Cochrane Database Syst Rev 2019;10:ED000142.

30. Hozo SP, Djulbegovic B, Hozo I. Estimating the mean and variance from the median, range, and the size of a sample. BMC Med Res Methodol 2005;5:13.

31. Sanguankeo A, Upala S, Cheungpasitporn W, et al. Effects of Statins on Renal Outcome in Chronic Kidney Disease Patients: A Systematic Review and Meta-Analysis. PLoS One 2015;10:e132970.

32. Review Manager (RevMan) [computer program]. Version 5.4.1. Copenhagen, Denmark: The Nordic Cochrane Centre, The Cochrane Collaboration; 2020.

33. Block GA, Fishbane S, Rodriguez M, et al. A 12-week, double-blind, placebo-controlled trial of ferric citrate for the treatment of iron deficiency anemia and reduction of serum phosphate in patients with CKD Stages 3-5. Am J Kidney Dis 2015;65:728-36.

34. Chue CD, Townend JN, Moody WE, et al. Cardiovascular effects of sevelamer in stage 3 CKD. J Am Soc Nephrol 2013;24:842-52.

35. Ureña-Torres P, Prié D, Keddad K, et al. Changes in fibroblast growth factor 23 levels in normophosphatemic patients with chronic kidney disease stage 3 treated with lanthanum carbonate: results of the PREFECT study, a phase 2a, double blind, randomized, placebo-controlled trial. BMC Nephrol 2014;15:71.

36. Iguchi A, Yamamoto S, Yamazaki M, et al. Effect of ferric citrate hydrate on FGF23 and PTH levels in patients with non-dialysis-dependent chronic kidney disease with normophosphatemia and iron deficiency. Clin Exp Nephrol 2018;22:789-96.

37. Otsuki T, Utsunomiya K, Moriuchi M, et al. Effect of Sucroferric Oxyhydroxide on Fibroblast Growth Factor 23 Levels in Hemodialysis Patients. Nephron 2018;140:161-8.

38. Isakova T, Barchi-Chung A, Enfield G, et al. Effects of dietary phosphate restriction and phosphate binders on FGF23 levels in CKD. Clin J Am Soc Nephrol 2013;8:1009-18.

39. Fishbane S, Block GA, Loram L, et al. Effects of Ferric Citrate in Patients with Nondialysis-Dependent CKD and Iron Deficiency Anemia. J Am Soc Nephrol 2017;28:1851-8.

40. Seifert ME, de las Fuentes L, Rothstein M, et al. Effects of phosphate binder therapy on vascular stiffness in early-stage chronic kidney disease. Am J Nephrol 2013;38:158-67.

41. Yokoyama K, Hirakata H, Akiba T, et al. Ferric citrate hydrate for the treatment of hyperphosphatemia in nondialysis-dependent CKD. Clin J Am Soc Nephrol 2014;9:543-52.

42. Block GA, Block MS, Smits G, et al. A Pilot Randomized Trial of Ferric Citrate Coordination Complex for the Treatment of Advanced CKD. J Am Soc Nephrol 
2019;30:1495-504.

43. Liabeuf S, Ryckelynck JP, El Esper N, et al. Randomized Clinical Trial of Sevelamer Carbonate on Serum Klotho and Fibroblast Growth Factor 23 in CKD. Clin J Am Soc Nephrol 2017;12:1930-40.

44. Phelps KR, Stote KS, Mason D. Use of sevelamer to examine the role of intraluminal phosphate in the pathogenesis of secondary hyperparathyroidism. Clin Nephrol 2014;82:191-201.

45. Chang YM, Tsai SC, Shiao CC, et al. Effects of lanthanum carbonate and calcium carbonate on fibroblast growth factor 23 and hepcidin levels in chronic hemodialysis patients. Clin Exp Nephrol 2017;21:908-16.

46. Lin HH, Liou HH, Wu MS, et al. Long-term sevelamer treatment lowers serum fibroblast growth factor 23 accompanied with increasing serum Klotho levels in chronic haemodialysis patients. Nephrology (Carlton) 2014;19:672-8.

47. Edmonston D, Wolf M. FGF23 at the crossroads of phosphate, iron economy and erythropoiesis. Nat Rev Nephrol 2020;16:7-19.

48. Isakova T, Gutiérrez OM, Smith K, et al. Pilot study of dietary phosphorus restriction and phosphorus binders to target fibroblast growth factor 23 in patients with chronic kidney disease. Nephrol Dial Transplant 2011;26:584-91.

49. Sprague SM, Abboud H, Qiu P, et al. Lanthanum carbonate reduces phosphorus burden in patients with CKD stages 3 and 4: a randomized trial. Clin J Am Soc Nephrol 2009;4:178-85.

50. Urakawa I, Yamazaki Y, Shimada T, et al. Klotho converts canonical FGF receptor into a specific receptor for FGF23. Nature 2006;444:770-4.

51. Goyal R, Jialal I. Hyperphosphatemia. StatPearls. Treasure Island (FL), 2021.

52. Sabbagh Y, Giral H, Caldas Y, et al. Intestinal phosphate transport. Adv Chronic Kidney Dis 2011;18:85-90.

Cite this article as: Zhao SJ, Wang ZX, Chen L, Wang FX, Kong LD. Effect of different phosphate binders on fibroblast growth factor 23 levels in patients with chronic kidney disease: a systematic review and meta-analysis of randomized controlled trials. Ann Palliat Med 2022;11(4):1264-1277. doi: 10.21037/apm21-1943
53. Agoro R, Ni P, Noonan ML, et al. Osteocytic FGF23 and Its Kidney Function. Front Endocrinol (Lausanne) 2020;11:592.

54. Shimada T, Hasegawa H, Yamazaki Y, et al. FGF-23 is a potent regulator of vitamin $\mathrm{D}$ metabolism and phosphate homeostasis. J Bone Miner Res 2004;19:429-35.

55. Marks J, Srai SK, Biber J, et al. Intestinal phosphate absorption and the effect of vitamin D: a comparison of rats with mice. Exp Physiol 2006;91:531-7.

56. Sabbagh Y, O'Brien SP, Song W, et al. Intestinal npt $2 \mathrm{~b}$ plays a major role in phosphate absorption and homeostasis. J Am Soc Nephrol 2009;20:2348-58.

57. Schiavi SC, Tang W, Bracken C, et al. Npt2b deletion attenuates hyperphosphatemia associated with CKD. J Am Soc Nephrol 2012;23:1691-700.

58. Isakova T, Ix JH, Sprague SM, et al. Rationale and Approaches to Phosphate and Fibroblast Growth Factor 23 Reduction in CKD. J Am Soc Nephrol 2015;26:2328-39.

59. Giral H, Caldas Y, Sutherland E, et al. Regulation of rat intestinal $\mathrm{Na}$-dependent phosphate transporters by dietary phosphate. Am J Physiol Renal Physiol 2009;297:F1466-75.

60. Ketteler M, Biggar PH. Use of phosphate binders in chronic kidney disease. Curr Opin Nephrol Hypertens 2013;22:413-20.

61. Susantitaphong P, Jaber BL. Potential interaction between sevelamer and fat-soluble vitamins: a hypothesis. Am J Kidney Dis 2012;59:165-7.

62. Perry CM, Plosker GL. Sevelamer carbonate: a review in hyperphosphataemia in adults with chronic kidney disease. Drugs 2014;74:771-92.

63. Swainston Harrison T, Scott LJ. Lanthanum carbonate. Drugs 2004;64:985-96; discussion 997-8.

64. Behets GJ, Verberckmoes SC, D'Haese PC, et al. Lanthanum carbonate: a new phosphate binder. Curr Opin Nephrol Hypertens 2004;13:403-9. 
Table S1 Literature search strategy

\section{PubMed/Medline}

\#1. $((()((()((()(((($ Renal Insufficiency, Chronic[Title/Abstract]) OR (Chronic Renal Insufficiencies[Title/Abstract])) OR (Renal Insufficiencies, Chronic[Title/Abstract])) OR (Chronic Renal Insufficiency[Title/Abstract])) OR (Kidney Insufficiency, Chronic[Title/Abstract])) OR (Chronic Kidney Insufficiency[Title/Abstract])) OR (Chronic Kidney Insufficiencies[Title/Abstract])) OR (Kidney Insufficiencies, Chronic[Title/Abstract])) OR (Chronic Kidney Diseases[Title/Abstract])) OR (Chronic Kidney Disease[Title/Abstract])) OR (Disease, Chronic Kidney[Title/Abstract])) OR (Diseases, Chronic Kidney[Title/Abstract])) OR (Kidney Disease, Chronic[Title/Abstract])) OR (Kidney Diseases, Chronic[Title/Abstract])) OR (Chronic Renal Diseases[Title/Abstract])) OR (Chronic Renal Disease[Title/Abstract])) OR (Disease, Chronic Renal[Title/Abstract])) OR (Diseases, Chronic Renal[Title/Abstract])) OR (Renal Disease, Chronic[Title/Abstract])) OR (Renal Diseases, Chronic[Title/Abstract])

\#2. (((()(((((ferric citrate[Title/Abstract]) OR (iron(III) citrate[Title/Abstract])) OR (ferric-citric acid[Title/Abstract])) OR (ferric citrate anhydrous[Title/Abstract])) OR (ferric citrate hydrate[Title/Abstract])) OR (JTT-751[Title/Abstract])) OR (zerenex[Title/Abstract])) OR (ferric citrate trihydrate[Title/Abstract])) OR (ferric citrate, 59Fe-labeled cpd[Title/Abstract])) OR (ferric citrate, iron salt, 59Fe-labeled cpd[Title/ Abstract])) OR (ferric citrate dihydrate[Title/Abstract])) OR (ferric citrate iron(+3) salt[Title/Abstract])

\#3. (((((((Sevelamer[Title/Abstract]) OR (GT335-012[Title/Abstract])) OR (GT335 012[Title/Abstract])) OR (GT335012[Title/Abstract])) OR (Sevelamer Hydrochloride[Title/Abstract])) OR (Hydrochloride, Sevelamer[Title/Abstract])) OR (RenaGel[Title/Abstract])) OR (Sevelamer Carbonate[Title/Abstract])) OR (Carbonate, Sevelamer[Title/Abstract])

\#4. ((Lanthanum Carbonate[Title/Abstract]) OR (lantharenol[Title/Abstract])) OR (Fosrenol[Title/Abstract])

\#5. \#2 OR \#3 OR \#4

\#6. ((((Fibroblast Growth Factor 23[Title/Abstract]) OR (FGF23 protein, human[Title/Abstract])) OR (fibroblast growth factor 23, human[Title/ Abstract])) OR (tumor-derived hypophophatemia inducing factor, human[Title/Abstract])) OR (FGF-23 protein, human[Title/Abstract])

\#7. \#1 AND \#5 AND \#6 


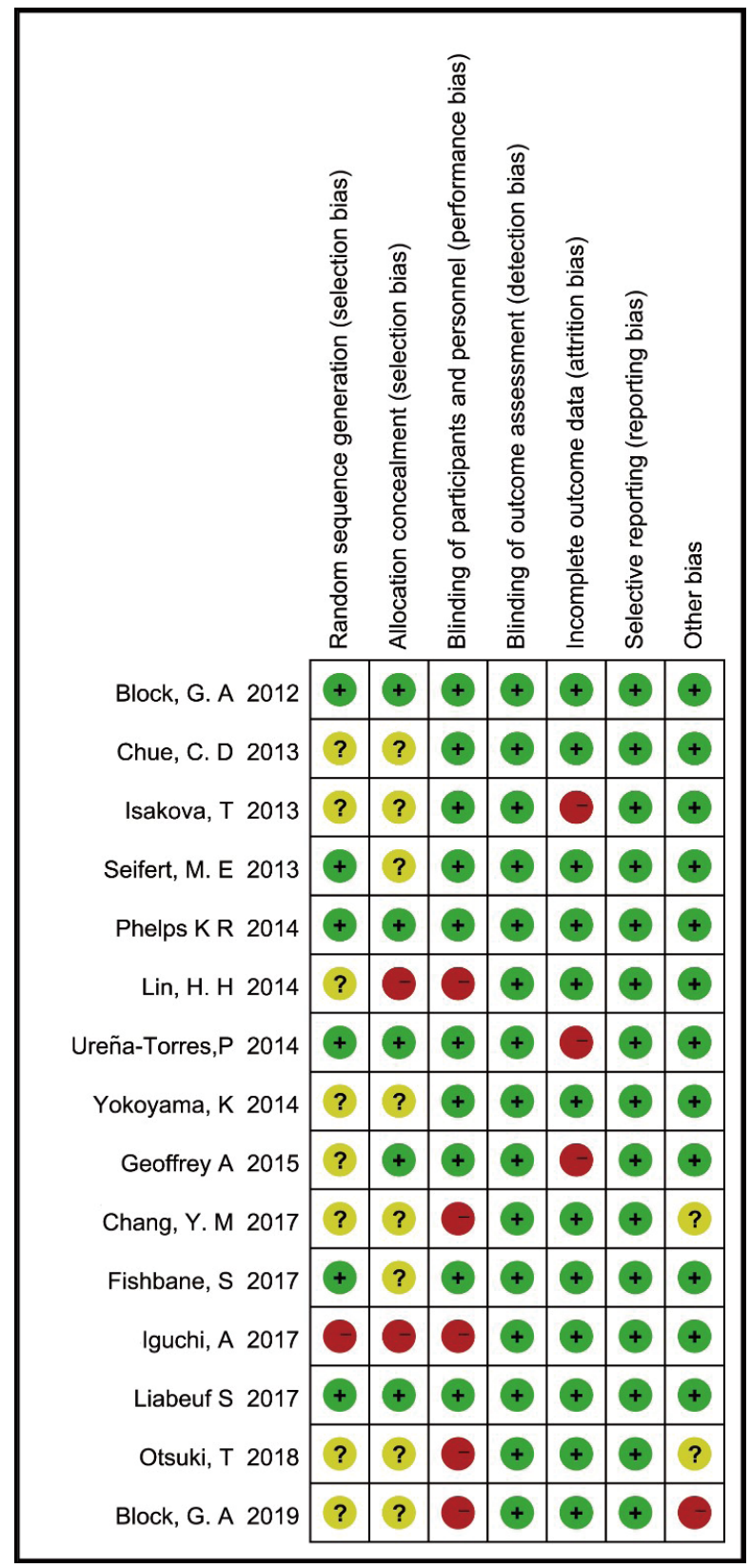

Figure S1 Risk of bias summary: each risk of bias item for each included study. 